\title{
Electron-impact vibrational excitation of cyclopropane
}

\author{
R. Čurík, ${ }^{1, a)}$ P. Čársky, ${ }^{1}$ and M. Allan ${ }^{2}$ \\ ${ }^{1}$ J. Heyrovský Institute of Physical Chemistry, Academy of Sciences of the Czech Republic, v.v.i., Dolejškova 3, \\ 18223 Prague 8, Czech Republic \\ ${ }^{2}$ Department of Chemistry, University of Fribourg, CH-1700 Fribourg, Switzerland
}

\begin{abstract}
We report a very detailed test of the ab initio discrete momentum representation (DMR) method of calculating vibrational excitation of polyatomic molecules by electron impact, by comparison of its results with an extensive set of experimental data, covering the entire range of scattering angles from $10^{\circ}$ to $180^{\circ}$ and electron energies from 0.4 to $20 \mathrm{eV}$. The DMR calculations were carried out by solving the two-channel Lippmann-Schwinger equation in the momentum space, and the interaction between the scattered electron and the target molecule was described by exact static-exchange potential corrected by a density functional theory (DFT) correlation-polarization interaction that models target's response to the field of incoming electron. The theory is found to quantitatively reproduce the measured spectra for all normal modes, even at the difficult conditions of extreme angles and at low energies, and thus provides full understanding of the excitation mechanism. It is shown that the overlap of individual vibrational bands caused by limited experimental resolution and rotational excitation must be properly taken into account for correct comparison of experiment and theory. By doing so, an apparent discrepancy between published experimental data could be reconciled. A substantial cross section is found for excitation of the non-symmetric HCH twisting mode $v_{4}$ of $A_{1}^{\prime \prime}$ symmetry by the $5.5 \mathrm{eV} A_{2}^{\prime}$ resonance, surprisingly because the currently accepted selection rules predict this process to be forbidden. The DMR theory shows that the excitation is caused by an incoming electron in an $f$-wave of $A_{2}^{\prime}$ symmetry which causes excitation of the non-symmetric $\mathrm{HCH}$ twisting mode $v_{4}$ of the $A_{1}^{\prime \prime}$ symmetry and departs in $p$ - and $f$-waves of $A_{2}^{\prime \prime}$ symmetry.
\end{abstract}

\section{INTRODUCTION}

This paper deals with the coupling of electronic and nuclear motions in temporary negative ions of polyatomic molecules, as revealed by the cross sections for vibrational excitation by electron impact. In a more general context, this subject is important for electron-driven chemistry with its many applications. Computational modeling of these inelastic processes for polyatomic molecules is not a resolved problem. There have been many rigorous and successful attempts for diatomic $^{1-4}$ and triatomic ${ }^{5-7}$ molecules but very few results are available for polyatomic systems. Our preliminary report on cyclopropane $^{8}$ indicated that the recently developed discrete momentum representation (DMR) theory with improved treatment of polarization represents a substantial progress in this direction and in this paper we present a comprehensive test of this theory and the insight which it provides.

We believe that at the present state a treatment of electronimpact vibrational excitation of polyatomic molecules requires numerically controlled approximations and the DMR theory applied here is of this type. It shows that it is possible to retain both the rigor of the theory and computational feasibility while applying such approximations. ${ }^{9-11}$

In contrast to modeling of vibrational excitation, the modeling of elastic collisions of electrons with polyatomic

${ }^{a)}$ Electronic mail: roman.curik@jh-inst.cas.cz molecules became a well-established task during the last two decades. Many authors report fixed-nuclear elastic data for the polyatomic molecules obtained by use of the R-matrix method, ${ }^{12,13}$ the complex Kohn variational method, ${ }^{14,15}$ the Schwinger multichannel method (SMC), ${ }^{16-18}$ or the optical potential method with single-center expansion of electron wave function. ${ }^{19,20}$ This paper, therefore, does not emphasize on elastic scattering, but some of it is also included since the capacity of the DMR method to describe vibrational excitation relies on its capacity to correctly reproduce the elastic cross sections.

The cyclopropane molecule was chosen as the test case because it represents a useful compromise between being sufficiently large and at the same time having a manageable number of vibrational modes. It also has a number of resonances which are narrow for a saturated hydrocarbon and thus permit convenient evaluation of the capacity of the theory to describe correctly the energies and widths of the resonances. A marked feature observed by experiments ${ }^{21}$ is a quite narrow shape resonance at $5.5 \mathrm{eV}$ of $\mathrm{A}_{2}^{\prime}$ symmetry, causing primarily excitation of the $v_{3}$ vibration, the $\mathrm{C}-\mathrm{C}$ ring stretching. These findings were confirmed computationally. ${ }^{22}$ In that study, the authors obtained a qualitative agreement of the resonance position (calculated too high by $1 \mathrm{eV}$ ) but the energy dependence of the cross section differed significantly from experiment above the resonant energy. An unresolved problem concerns a shape resonance reported 
experimentally at $2.6 \mathrm{eV},{ }^{21,23}$ which has so far not been reproduced by theory. ${ }^{24}$ The cyclopropane molecule is also of practical interest; it was detected in cooler edges of the fusion plasmas. ${ }^{25}$

The aim of the present study is twofold. It reports a comprehensive test of the DMR theory against experiment. Our earlier work ${ }^{8}$ successfully tested selected aspects of the DMR theory - an energy-loss spectrum at an incident energy of $5.5 \mathrm{eV}$, one case of an angular distribution and two cases of cross section recorded as a function of electron energy. To ascertain that the good agreement found in that study was not fortuitous, we present here a comprehensive comparison, at electron energies in the range 1.0-20 eV and a wide range of scattering angles. Particular attention is paid to problems arising from the overlap of vibrational bands due to their proximity and the limited instrumental resolution.

The second aim is to apply the theory, once validated by the detailed comparison with experiment, to illuminate the detailed properties of the resonances. It will be shown that the present theory provides a detailed insight into the mechanism of excitation, particularly of the non-totally symmetrical vibrations. Our results are more general and go beyond those of the symmetry selection rules, originally formulated by Wong and Schulz ${ }^{26}$ and later elaborated by Gallup ${ }^{27}$ who based his finding on theoretical grounds by using the Feshbach-Fano partitioning technique.

\section{EXPERIMENT}

The experimental conditions of the present work were the same as in our earlier study ${ }^{8}$ but a much larger body of data is reported. Altogether, 24 energy-loss spectra normalized to absolute values were measured at the scattering angles of $45^{\circ}, 90^{\circ}, 135^{\circ}, 180^{\circ}$ and incident electron energies of 1.0 , 2.6, 5.5, 10, 15, and $20 \mathrm{eV}$. Selected cross sections were further measured as a function of electron energy at $45^{\circ}, 90^{\circ}$, $135^{\circ}$, and $180^{\circ}$. The elastic cross section was measured as a function of electron energy at $45^{\circ}, 90^{\circ}, 135^{\circ}$, and $180^{\circ}$ and as a function of scattering angle at 1.0, 2.6, 5.5, 10, 15, and $20 \mathrm{eV}$. As in the previous study, ${ }^{8}$ the technical quality of the data is substantially improved in comparison with the older measurements ${ }^{21,23}$ in terms of resolution, extended angular range made possible by the magnetic angle changer (MAC), and accuracy of the absolute values.

Briefly, the measurements were performed with a spectrometer using hemispherical analyzers. ${ }^{28,29}$ Absolute values of the cross sections were determined by the relative flow technique and normalized to a theoretical helium elastic cross section. The two-standard-deviation confidence limit for the magnitudes of the inelastic cross sections is about $\pm 25 \%$. The angular distributions were measured using combined mechanical setting of the analyzer and magnetic deflection using a magnetic angle changer. The resolution was about $15 \mathrm{meV}$ in the energy-loss mode.

Integral elastic cross sections were derived by integrating under the angular distributions. A narrow range around $0^{\circ}$, where elastic cross section cannot be measured, was obtained by visual extrapolation.

\section{DISCRETE MOMENTUM REPRESENTATION}

The DMR method is a rigorous ab initio method ${ }^{30,31}$ based on the two-channel Lippmann-Schwinger equation. For actual calculations, we used the following numerically controllable approximations:

- We introduce one-electron optical potential $V$ for the interaction between the scattered electron and the charge density of the molecule. Moreover, we retain only the first term of the optical potential expansion, ${ }^{32}$ ending up with the static-exchange (SE) approximation.

- The SE approximation is corrected by a model density functional theory (DFT) polarization potential $V_{\mathrm{cp}}$ that accounts for orbital relaxation of the bound electrons and for the correlation between the scattered electron and the bound electrons. We used the interpolation formula suggested by Perdew and Zunger. ${ }^{33}$

- Nuclear dynamics is described by the rotationally frozen and vibrationally harmonic approximations. Moreover, for the vibrational space of each normal mode, we use only a two-state approximation.

As a result of this approximation, the two-channel Lippmann-Schwinger equation for a transition operator $T$ may be written as

$$
\begin{aligned}
\left\langle\chi_{1} \boldsymbol{k}_{1}|T| \chi_{0} \boldsymbol{k}_{0}\right\rangle= & \left\langle\chi_{1} \boldsymbol{k}_{1}|U| \chi_{0} \boldsymbol{k}_{0}\right\rangle \\
& +\sum_{i=0}^{1} \int \mathrm{d} \boldsymbol{k} \frac{\left\langle\chi_{1} \boldsymbol{k}_{1}|U| \chi_{i} \boldsymbol{k}\right\rangle\left\langle\chi_{i} \boldsymbol{k}|T| \chi_{0} \boldsymbol{k}_{0}\right\rangle}{k_{0}^{2}-2 E_{i}-k^{2}+\mathrm{i} \varepsilon},
\end{aligned}
$$

where $U$ stands for a double of the interaction potential $V, E_{0}=0$ (for the elastic channel) is the energy of the vibrational ground state, and $E_{1}$ is the energy of the first excited vibrational state. These two states are described by the harmonic vibrational functions $\chi_{0}$ and $\chi_{1}$, respectively. The vectors $\boldsymbol{k}_{0}$ and $\boldsymbol{k}_{1}$ represent the plane-wave functions for the incoming and outgoing electrons, respectively.

A numerical discretization of the integral on rhs of the above equation leads to a set of two coupled matrix equations,

$$
\begin{aligned}
{\left[\begin{array}{ll}
T_{00} & T_{01} \\
T_{10} & T_{11}
\end{array}\right]=} & {\left[\begin{array}{ll}
U_{00} & U_{01} \\
U_{10} & U_{11}
\end{array}\right]+\left[\begin{array}{ll}
U_{00} & U_{01} \\
U_{10} & U_{11}
\end{array}\right] } \\
& \times\left[\begin{array}{ll}
G_{0} & \\
& G_{1}
\end{array}\right]\left[\begin{array}{ll}
T_{00} & T_{01} \\
T_{10} & T_{11}
\end{array}\right]
\end{aligned}
$$

with the following interaction matrix elements:

$$
\begin{aligned}
& {\left[U_{00}\right]_{i j}=\left\langle\chi_{0} \boldsymbol{k}_{i}|U| \chi_{0} \boldsymbol{k}_{j}\right\rangle=\left[U_{11}\right]_{i j},} \\
& {\left[U_{01}\right]_{i j}=\left\langle\chi_{0} \boldsymbol{k}_{i}|U| \chi_{1} \boldsymbol{k}_{j}\right\rangle=\left[U_{10}\right]_{i j} .}
\end{aligned}
$$

The above identities are based on a first order expansion of the interaction along the dimensionless normal mode coordinate $Q$ as

$$
U(Q)=U(0)+Q \frac{\partial}{\partial Q} U(0)+O\left(Q^{2}\right)
$$

where $U(0)$ stands for the double of interaction potential at equilibrium geometry. Neglecting the higher order terms 
$O\left(Q^{2}\right)$ results in a simple integration over the coordinate $Q$,

$$
\begin{aligned}
& \left\langle\chi_{0}(Q) \boldsymbol{k}_{i}|U(Q)| \chi_{0}(Q) \boldsymbol{k}_{j}\right\rangle=\left\langle\boldsymbol{k}_{i}|U(0)| \boldsymbol{k}_{j}\right\rangle, \\
& \left\langle\chi_{0}(Q) \boldsymbol{k}_{i}|U(Q)| \chi_{1}(Q) \boldsymbol{k}_{j}\right\rangle=\frac{1}{\sqrt{2}} \frac{\partial}{\partial Q}\left\langle\boldsymbol{k}_{i}|U(0)| \boldsymbol{k}_{j}\right\rangle .
\end{aligned}
$$

The interaction potential $V(Q)=U(Q) / 2$ is generated from target state electron density

$$
\rho(Q)=\sum_{\mu \nu} D_{\mu v}(Q) g_{\mu}(Q) g_{v}(Q),
$$

where $D_{\mu \nu}(Q)$ is a Hartree-Fock density matrix and $g_{\mu}(Q)$ represents Gaussian type basis set. As already mentioned, the interaction potential $V(Q)$ consists of static, exchange and correlation-polarization contributions

$$
V(Q)=V_{\mathrm{s}}(Q)+V_{\mathrm{ex}}(Q)+V_{\mathrm{cp}}(Q) .
$$

For simplicity in the following formulas, we suppress explicit dependence on vibrational coordinate $Q$. Momentum-space matrix elements (5) of $V_{\mathrm{s}}$ and $V_{\mathrm{ex}}$ are then evaluated through hybrid plane-wave and Gaussian repulsion integrals

$$
\begin{aligned}
\left\langle\boldsymbol{k}\left|V_{\mathrm{s}}\right| \boldsymbol{q}\right\rangle= & \sum_{\mu \nu} D_{\mu \nu}\left(g_{\mu} g_{v} \mid \boldsymbol{k} \boldsymbol{q}\right) \\
& -\frac{1}{2 \pi^{2}|\boldsymbol{q}-\boldsymbol{k}|^{2}} \sum_{A=1}^{M} Z_{A} e^{\mathrm{i}(\boldsymbol{q}-\boldsymbol{k}) \cdot \boldsymbol{R}_{A},} \\
\left\langle\boldsymbol{k}\left|V_{\mathrm{ex}}\right| \boldsymbol{q}\right\rangle= & -\sum_{\mu \nu} D_{\mu \nu}\left(\boldsymbol{k} g_{\mu} \mid g_{\nu} \boldsymbol{q}\right),
\end{aligned}
$$

with the second term of Eq. (9) representing a nuclear attraction to $M$ nuclei positioned at vectors $\boldsymbol{R}_{A}$. The static and exchange repulsion integrals are defined as follows:

$$
\begin{aligned}
& \left(g_{\mu} g_{\nu} \mid \boldsymbol{k} \boldsymbol{q}\right)=\frac{1}{(2 \pi)^{3}} \iint \mathrm{d} \boldsymbol{x} \mathrm{d} \boldsymbol{y} \frac{g_{\mu}(\boldsymbol{x}) g_{\nu}(\boldsymbol{x}) e^{\mathrm{i}(\boldsymbol{q}-\boldsymbol{k}) \cdot \boldsymbol{y}}}{|\boldsymbol{x}-\boldsymbol{y}|}, \\
& \left(\boldsymbol{k} g_{\mu} \mid g_{\nu} \boldsymbol{q}\right)=\frac{1}{(2 \pi)^{3}} \iint \mathrm{d} \boldsymbol{x} \mathrm{d} \boldsymbol{y} \frac{g_{\mu}(\boldsymbol{x}) e^{-\mathrm{i} \boldsymbol{k} \cdot \boldsymbol{x}} g_{\nu}(\boldsymbol{y}) e^{\mathrm{i} \boldsymbol{q} \cdot \boldsymbol{y}}}{|\boldsymbol{x}-\boldsymbol{y}|} .
\end{aligned}
$$

In most of the present implementations of a correlation potential in quantum chemistry, the $V_{\mathrm{cp}}$ is calculated as a function of the electron density $\rho$ and its spatial gradient $\nabla \rho$. Similarly, we evaluate $V_{\text {cp }}$ on a rectangular spatial grid with the molecule sitting in its center. The incorrect long-range behavior of $V_{\mathrm{cp}}$, so common in DFT modeling, is then corrected by a polarization asymptotic potential. ${ }^{34}$ The required polarizabilities are obtained by $a b$ initio quantum chemistry software as described below in this section. The momentum space matrix elements are computed by use of fast Fourier transform (FFT) technique applied on the following integral:

$$
\left\langle\boldsymbol{k}\left|V_{\mathrm{cp}}\right| \boldsymbol{q}\right\rangle=\frac{1}{(2 \pi)^{3}} \int \mathrm{d} \boldsymbol{r} V_{\mathrm{cp}}(\boldsymbol{r}) e^{\mathrm{i}(\boldsymbol{q}-\boldsymbol{k}) \cdot \boldsymbol{r}} .
$$

Convergence of the above integral with respect to FFT box size is quite slow owing to the long-range nature of $V_{\mathrm{cp}}$. However, the asymptotic tail of $V_{\mathrm{cp}}$ may be removed and evaluated analytically ${ }^{34}$ leaving a short-range part of $V_{\mathrm{cp}}$ that is amenable to the numerical evaluation based on FFT. Vibrational coupling elements (6) require differentiation of Eqs. (9)-(13) with respect to coordinates of the nuclei. The nuclear gradients are implemented in the DMR method in analytical form. ${ }^{7}$
Electronic state of the target molecule is described at the Hartree-Fock level. We used Gaussian type orbital basis set of the DZP quality. ${ }^{35}$ Polarizability tensor components used in the present calculations were obtained as linear response functions in Kohn-Sham DFT calculations ${ }^{36}$ with B3LYP hybrid functional and Sadlej's polarized VTZ basis sets $^{37}$ as implemented in program Dalton Release 2.0 (2005). The axes are defined to diagonalize the polarizability tensor, with diagonal polarizabilities $38.8,38.8$, and 33.8 a.u.; the average of these values, 37.1 a.u., compares favorably with the experimentally measured value of spherical polarizability $\alpha_{0}=38.2$ a.u. ${ }^{38}$ Evaluation of vibrational coupling elements (6) also requires gradients of the above tensor with respect to nuclear positions. These were obtained by numerical differentiation with shifting nuclei out of the equilibrium by $h=0.001$ bohr.

\section{COMPARISON OF MEASURED AND CALCULATED CROSS SECTIONS}

\section{A. Elastic cross sections}

Although elastic scattering is not of our primary interest in this paper, it is prudent to check whether our elastic data agree well with available calculations and experiments because the accuracy of our vibrationally inelastic cross sections depends on the accuracy of the elastic results - the two channels are coupled via Eq. (2). We find satisfactory agreement both for the integral elastic cross section plotted as a function of electron energy shown in Fig. 1 (for collision energies below $10 \mathrm{eV}$ ) and for the differential elastic cross section at $5.5 \mathrm{eV}$, displayed in Fig. 2. The differential cross sections were also measured at $0.4,1.0,2.6,10,15$, and $20 \mathrm{eV}$ and compare favorably to the present and earlier calculations as shown in supplementary ${ }^{44}$ figures S1 and S2. Disagreement between theory and experiment for energies above $10 \mathrm{eV}$

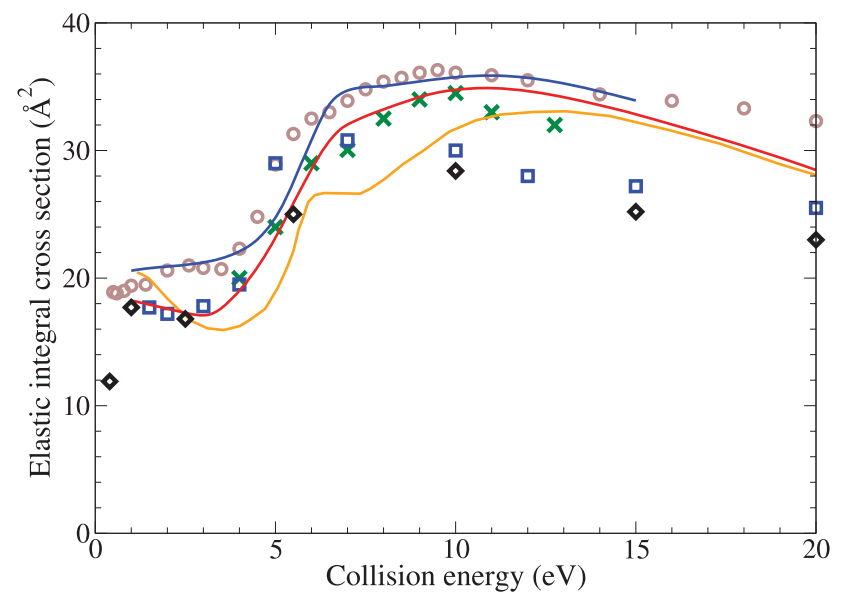

FIG. 1. Comparison of the elastic integral cross sections. Previous elastic measurements are shown with crosses ${ }^{39}$ and squares, ${ }^{40}$ while circles represent the previously reported total cross section. ${ }^{41}$ Present experimental data are denoted by diamonds. Present calculations are shown as red line, while the orange line represents previous $\mathrm{SMC},{ }^{40}$ and blue line represents results of single-center expansion method. ${ }^{42}$ The oldest SMC results ${ }^{43}$ are omitted here for clarity reasons. 


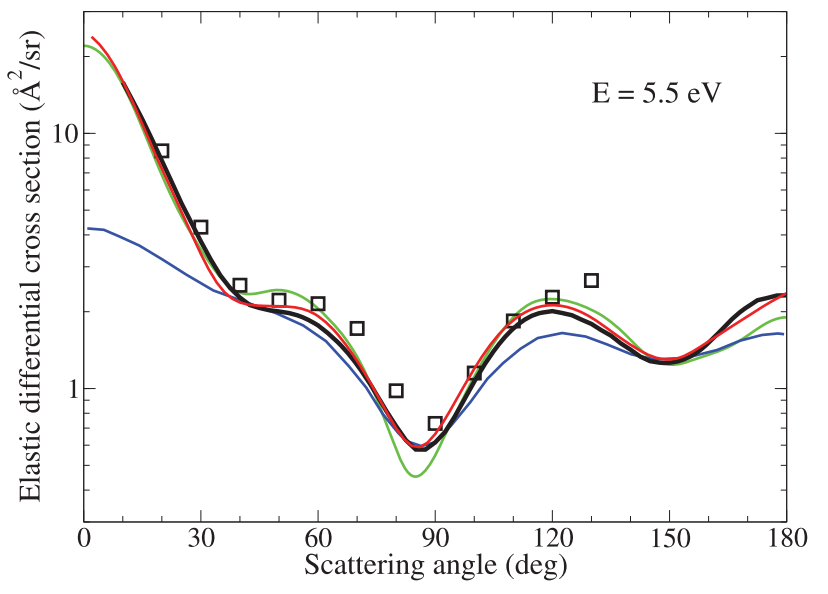

FIG. 2. Comparison of the elastic differential cross sections at $5.5 \mathrm{eV}$. The present experimental data are shown with the black line, while the previous measurements are denoted by squares. ${ }^{40}$ The red line shows present calculations, blue line displays results of previous Schwinger multi-channel calculations, ${ }^{40}$ and the green line represents single-center expansion calculations. ${ }^{42}$

may be assigned to the use of a single (electronic) channel model. Since the ionization potential of cyclopropane is at about $10 \mathrm{eV}^{45}$ and the lowest excited electronic states start at about $6.3 \mathrm{eV},{ }^{46}$ multiple electronic channels, not accounted for by single channel approach, become open. On the other hand, this limitation may increase confidence in the theory that its excellent performance at lower energy is not fortuitous.

The elastic cross section may also provide hints about the presence and positions of shape resonances, although for polyatomic molecules, the effect is often visible only weakly on the background of direct scattering. Humps indicative of resonances are visible around $6-7 \mathrm{eV}$ in the earlier theoretical integral cross sections displayed in Fig. 1 (orange and blue lines) but are not convincingly reproduced by the present theory and the experiments. More evidence for a resonance is provided by the strong $f$-wave character of the differential cross section at $5.5 \mathrm{eV}$, displayed in Figure 2.

\section{B. Cross sections for vibrational excitation}

\section{Electron energy-loss (EEL) spectra normalized to absolute values}

For polyatomic molecules, a detailed comparison between theory and experiment is hindered by the fact that the experiment suffers from overlap of close-lying vibrational peaks, caused mainly by the limited instrumental resolution, but also by the finite rotational temperature that broadens the vibrational peaks. This means that it is not possible to obtain an experimental cross section, plotted as a function of scattering angle or as a function of electron energy, for a single vibrational mode, without contributions of nearby partially overlapping bands. We by-pass this problem primarily by comparing EEL spectra instead of the more conventional comparison of the differential cross section for a given vibrational mode plotted as a function of scattering angle or electron energy. We compare the experimental spectra with profiles obtained by convoluting the calculated bar spectrum by a simulated experimental profile, a Gaussian of $15 \mathrm{meV}$

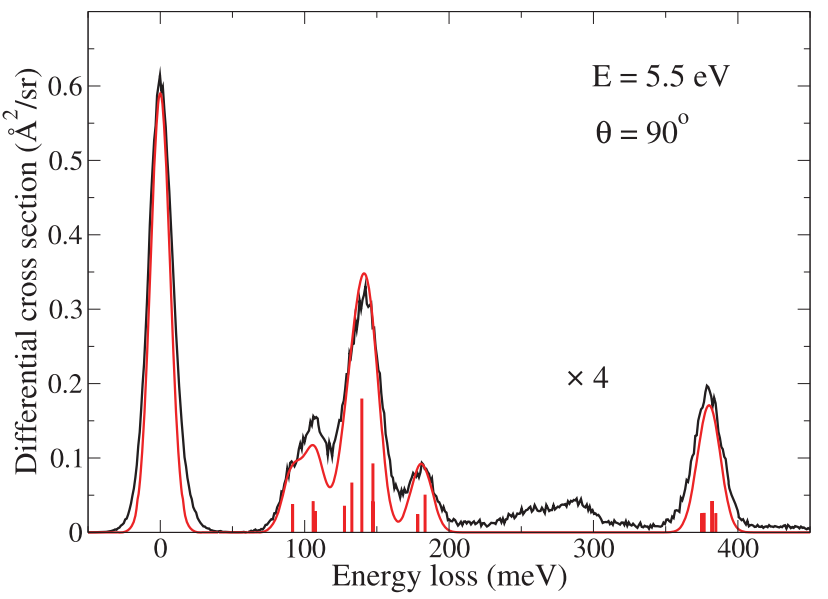

FIG. 3. Computed and measured absolute electron energy-loss spectra for the scattering angle $\vartheta=90^{\circ}$. Red line shows the present calculations while the black line denotes the experimental spectrum. Collision energy is $5.5 \mathrm{eV}$. The scale factor $\times 4$ applies only to inelastic bands.

width. An additional advantage of comparing EEL spectra is that it verifies all vibrational modes, because when the cross section is not calculated properly, the profiles will not fit even though the vibrational modes are not fully resolved.

A comprehensive test of the DMR theory against experiment requires a comparison for wide ranges of scattering angles and electron energies, as well as for all vibrational modes. We, therefore, measured the electron energy-loss spectra, normalized to absolute values, at the scattering angles of $45^{\circ}$ to $90^{\circ}, 135^{\circ}$ and $180^{\circ}$ and incident electron energies of $1.0,2.6,5.5,10,15$, and $20 \mathrm{eV}$. Two representative spectra are shown in Figures 3 and 4, the remaining spectra (24 spectra altogether) are shown in supplementary ${ }^{44}$ figures S3, S4, and S5.

It can be seen that agreement between theory and the experiment is very good for all the scattering angles and the collision energies up to $10 \mathrm{eV}$. However, above $10 \mathrm{eV}$, we observe that the theory predicts higher intensities over all the vibrational bands. We believe that the reason behind this

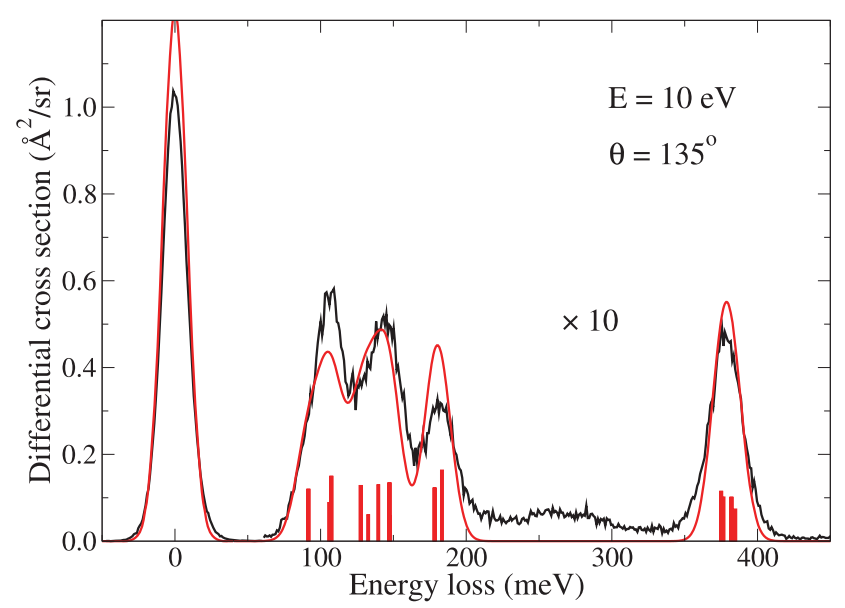

FIG. 4. Computed and measured absolute electron energy-loss spectra for the scattering angle $\vartheta=135^{\circ}$ and collision energy of $10 \mathrm{eV}$. Red line shows the present calculations while the black line denotes the experimental spectrum. The scale factor $\times 10$ applies only to inelastic bands. 
overestimate is the higher elastic cross section predicted by theory for collision energies above $10 \mathrm{eV}$ (see Fig. 1). Since the elastic and inelastic channels are coupled in the present theory, the higher elastic flux often causes also an artificial increase in the inelastic channel.

We would like to emphasize that no scaling of data was made in any of the EELS graphs. Both sets of data, the experiment and the theory, are compared in absolute values. Vertical lines, placed at the respective vibrational frequencies, denote magnitudes of the calculated differential cross sections. We also note that the bands observed experimentally at 220-320 meV correspond to overtone excitations and are therefore not amenable to treatment by harmonic approximation inherent in the DMR model.

\section{Dependence of cross sections on electron energy}

Resonances appear as enhancements of vibrational cross sections over narrow ranges of energies. Plots of cross sections, as a function of electron energy, thus represent a sensitive test of the capacity of the DMR theory to describe the energies and widths of resonances.

The calculated vibrational excitation cross sections for all 14 vibrational modes are displayed in Figure 5, ordered by the increasing energy loss. The results are compared with previous calculations ${ }^{22}$ for the three fully symmetric modes $v_{1}, v_{2}$, and $v_{3}$. We note that in the case of the $v_{1}$ mode the two computations agree well, present calculations being slightly higher in the resonant region. The difference is larger for $v_{3}$. For $v_{2}$, the agreement is good above about $5 \mathrm{eV}$, but the present calculation yields a distinct resonant peak at $2.6 \mathrm{eV}$ which is missing in the previous calculation.
The resonance at $2.6 \mathrm{eV}$ deserves a special mention. Originally, it was assigned to the $A_{2}^{\prime \prime}$ pseudo- $\pi$ resonance, on the grounds of experimental finding, ${ }^{23}$ based on the selective excitation of the $\mathrm{CH}_{2}$ scissoring normal mode. It has the particularity of being much lower in energy than what is generally reported as the lowest shape resonance in saturated hydrocarbons where the vibrational excitation cross sections peak around $8 \mathrm{eV}^{21,23}$ (Saturated hydrocarbons do not have low-lying $\pi^{*}$ orbitals which give rise to low-lying shape resonances in compounds as ethene or benzene.) Finding this resonance at such a low energy helps to understand electron transfer through saturated alkyl bridges ${ }^{47,48}$ because it is related to the "empty state" giving rise to the conduction band. Early R-matrix calculations ${ }^{24}$ failed to reproduce this resonance. The more recent calculation ${ }^{22}$ yields a weak hump at $3.7 \mathrm{eV}$, which was, however, seen in the $A_{1}^{\prime}$ symmetry, in contrast to the assignment derived experimentally. A direct comparison between the present theory and experiment, confirming the presence of the $A_{2}^{\prime \prime}$ resonance, was already published $^{8}$ and we avoid further analysis of the $A_{2}^{\prime \prime}$ resonance here.

In case of the $v_{3}$ mode, our calculated data place the $5.5 \mathrm{eV}$ shape resonance at $6.0 \mathrm{eV}$ while previous calculations ${ }^{22}$ placed it at $6.4 \mathrm{eV}$. Note that many scattering theories predict shape resonances too high because of lack of, or insufficient, polarization of the target. Compared to previous calculations, ${ }^{22}$ we predict a somewhat lower energy and the cross section about twice as high. This indicates that the present version of the DMR theory accounts for polarization somewhat better. This point will be elaborated later.

The dominant feature of the excitation cross sections summarized in Figure 5 is the $5.5 \mathrm{eV}$ shape resonance that

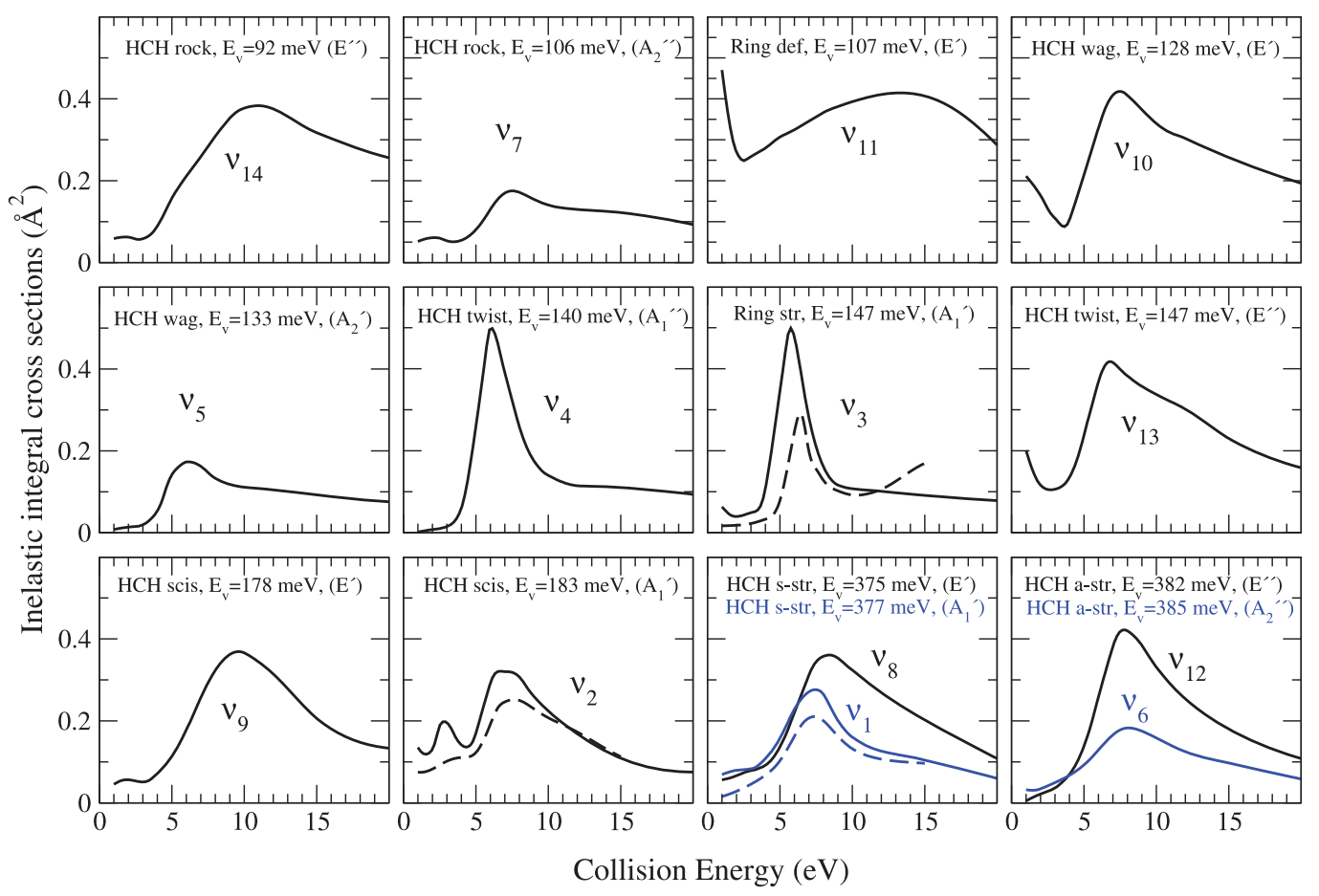

FIG. 5. Calculated rotationally summed $0 \rightarrow 1$ vibrationally inelastic integral cross sections (full lines). The modes are ordered by increasing vibrational frequency. Our results are compared to previous calculations ${ }^{22}$ shown with dashed lines. Each of the last two graphs contains two modes for space saving purposes. 


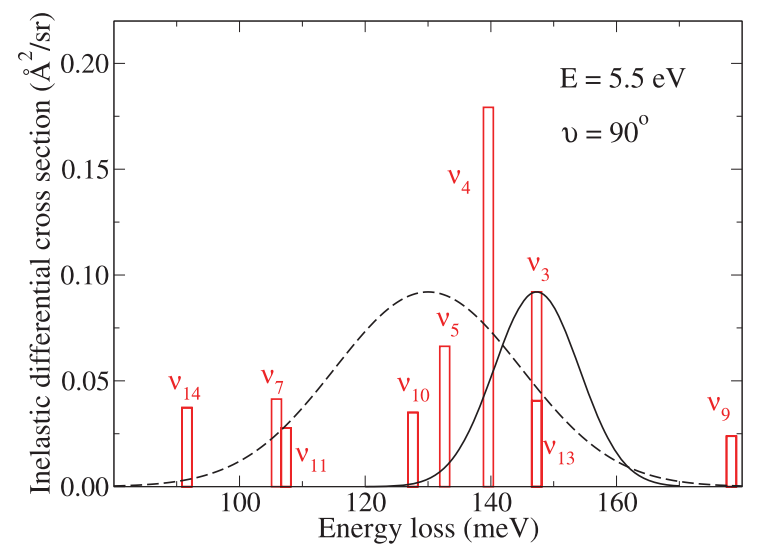

FIG. 6. Zoom of the line spectrum from Fig. 3 with two experimental resolution functions. The full line describes the present experiment, measured at an energy loss of $147 \mathrm{meV}$ and FWHM of $15 \mathrm{meV}$. The broken line denotes the experiment of Refs. 50 and 51 measured at an energy loss of $130 \mathrm{meV}$ with FWHM of $35 \mathrm{meV}$. The vertical bars indicate the calculated differential cross sections.

strongly influences the excitation of the $v_{3}$ and $v_{4}$ modes and also causes a weak maximum for the $v_{5}$ mode. The fact that we are speaking about one resonance influencing several modes will be proven in Sec. V. This resonance was first observed experimentally ${ }^{49}$ at $5.5 \mathrm{eV}$ and it was attributed to the $\mathrm{A}_{2}^{\prime}$ symmetry with arguments based on the mode selectivity of the vibrational excitation together with symmetry and nodal properties of virtual orbitals. ${ }^{21}$ This assignment was later confirmed by scattering calculations. ${ }^{22,43}$

As already pointed out, the cross sections of Figure 5 cannot be directly compared to the experiment, which necessarily samples several vibrations due to limited instrumental resolution and rotational broadening. In fact, there are two experiments for the nominally $v_{3}$ cross section, ${ }^{8,50}$ which seemingly contradict each other. We elaborate this point in detail on the supposed excitation of the $v_{3}$ vibration as shown in Fig. 6. (Note that $v_{3}$ generally dominates over $v_{4}$ for VE at $5.5 \mathrm{eV}$. It is only at scattering angles around $90^{\circ}$ as in Figure 6, where the excitation of $v_{3}$ has a minimum and that of $v_{4}$ has a maximum, that $v_{4}$ appears stronger than $v_{3}$.)

We take into account that the measured cross section is due to excitation of several near-lying normal modes as shown in Figure 6, a zoomed part of Figure 4, where we added two resolution functions describing the two available experiments. ${ }^{8,50}$ In the present experiment (and Ref. 8), the electrons were gathered at the nominal energy loss of the $v_{3}$ vibration, $147 \mathrm{meV}$, with a resolution [full width at half maximum (FWHM)] of $15 \mathrm{meV}$ (full line). In the second independent experiment, the energy loss was set to $130 \mathrm{meV}$ and the authors reported a resolution of $35 \mathrm{meV}$ (displayed by a dashed line in Fig. 6). In the following, we shall assume that the resolution functions do not depend on the collision energy in the range of 1-20 eV. In Figure 7, we show differential cross section for $0 \rightarrow 1$ vibrational excitation of all the 8 modes contributing to the signal of the second experiment, while only 4 of them $\left(v_{3}, v_{4}, v_{5}, v_{13}\right)$ are needed to reconstruct the first measurement. Figure 8 shows the weighted sums of cross sections for different modes with weights derived from positions and widths of the resolution functions displayed in

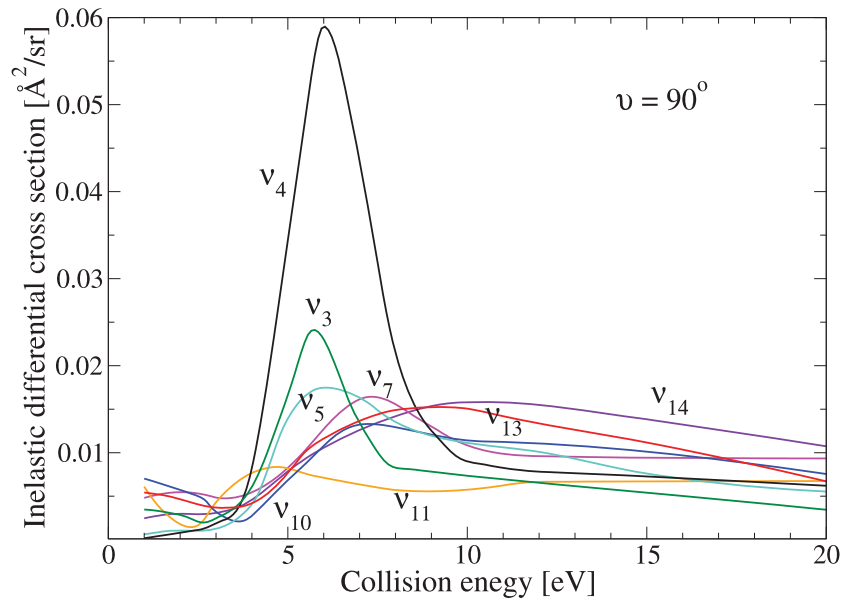

FIG. 7. Inelastic differential cross section as a function of collision energy for all 8 vibrational modes that contribute in reconstruction of experiments. ${ }^{8,50,51}$ Scattering angle is fixed at $\vartheta=90^{\circ}$.

Fig. 6. The weighted sums of the calculated data lead to a very good agreement with both experimental results and explain the seeming discrepancy between them: A wider resolution function gives electron current that contains probability from excitation of more vibrational modes, resulting in a higher value of the cross section.

Finally, it is worth noting that the broad shoulder in the experimental results visible in Fig. 8 around $10 \mathrm{eV}$ can be explained by the excitation of the $v_{13}$ mode that contributes to the measured signal in both experiments (see Figs. 6 and 7).

\section{SYMMETRY ANALYSIS OF VIBRATIONAL EXCITATION VIA THE 5.5 EV SHAPE RESONANCE}

The aim of this section is to determine computationally the dominant electronic symmetry components that drive resonant vibrational excitation at collision energy of $5.5 \mathrm{eV}$. Figure 5

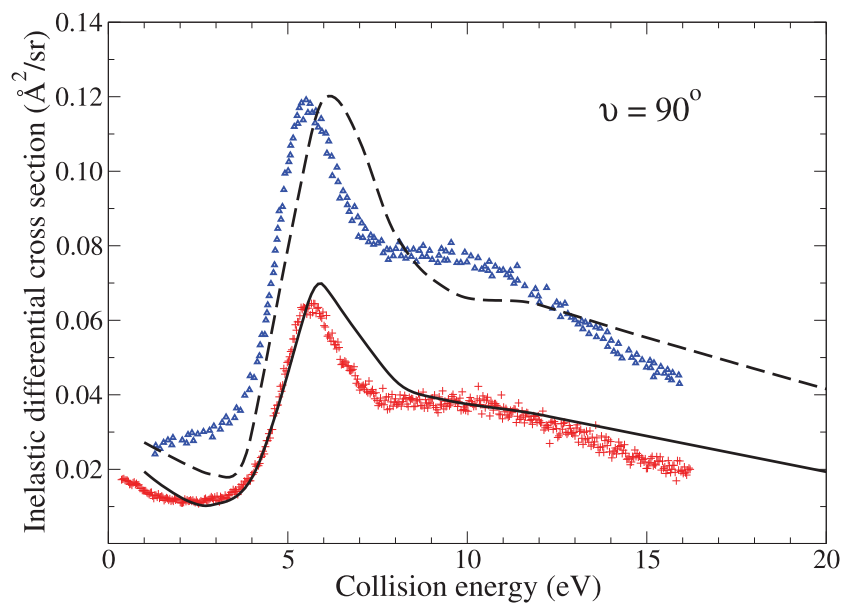

FIG. 8. Inelastic differential cross section as a function of collision energy. The two experiments are denoted by red crosses ${ }^{8}$ and blue triangles..$^{50,51}$ The full line represents a weighted sum of calculated inelastic cross sections for modes $v_{3}, v_{4}, v_{5}, v_{13}$; the broken line contains weighted contributions of all the 8 modes displayed in Fig. 7, with the weights reflecting the two instrumental profiles shown in Fig. 6. 
shows that there are two vibrational modes exhibiting strong resonant behavior at $5.5 \mathrm{eV}$, namely, the ring stretch mode $v_{3}$ and $\mathrm{HCH}$ twist mode $v_{4}$. One may argue that there is also a resonant enhancement of $\mathrm{HCH}$ wagging mode $v_{5}$; however, we find it very weak and hence it will not be in our focus.

At present, the DMR method does not attempt to reduce computational effort by making use of the symmetry components corresponding to the point group of the molecule. We have two reasons for this. First, the method is aimed at larger molecular systems that generally have low symmetry, and the computational gain would be small. Second, and more importantly, even symmetric molecules (as cyclopropane) reduce their symmetry if vibrational motion is included. We may work within two possible schemes: We may reduce the applied point group so that it is conserved for the particular vibrational mode or we may use the equilibrium point group ( $\mathrm{D}_{3 \mathrm{~h}}$ in this case) and accept that irreducible representations may become coupled by the vibrational motion. In the present analysis, we have chosen the latter.

We start with the $T_{10}$ matrix elements obtained by solving the set of equations (2),

$$
\left[T_{10}\right]_{i j}=\left\langle\chi_{1} \boldsymbol{k}_{i}|T| \chi_{0} \boldsymbol{k}_{j}\right\rangle
$$

where vectors $\boldsymbol{k}_{j}$ span the incoming momentum sphere with radius determined by collision energy $E$, and the vectors $\boldsymbol{k}_{i}$ span the outgoing momentum sphere with radius determined by energy of the outgoing electron. Since the angular grid on the sphere is described by Lebedev angular quadrature designed to integrate exactly spherical harmonics up to a particular order, ${ }^{52}$ the partial-wave components of the $T_{10}$ matrix are obtained by the straightforward technique

$$
T_{l m}^{l^{\prime} m^{\prime}}=\sum_{i j} w_{i} w_{j} S_{l m}\left(\hat{\boldsymbol{k}}_{i}\right)\left[T_{10}\right]_{i j} S_{l^{\prime} m^{\prime}}\left(\hat{\boldsymbol{k}}_{j}\right)
$$

where $w_{i}$ are the weights of the Lebedev quadrature, ${ }^{52}$ the vectors $\hat{\boldsymbol{k}}_{i}$ are unit vectors with directions of $\boldsymbol{k}_{i}$, and the angular functions $S_{l m}$ are chosen as real spherical harmonics. For a given orientation of the cyclopropane molecule in the $x y$-plane, the real spherical harmonics may be transformed by a real unitary transformation into angular functions that belong to an irreducible representation of the $\mathrm{D}_{3 \mathrm{~h}}$ point group. If we place one of the carbon atoms on the $y$-axis, this unitary transformation is very simple as the real spherical harmonics already form irreducible subspaces. ${ }^{53}$

Before we proceed to the analysis of the calculated symmetry components, we summarize the resonant symmetry selection rules first published by Wong and Schulz ${ }^{26}$ and later confirmed by Gallup. ${ }^{27}$ The rules were inspired by a very high resolution energy-loss spectrum of benzene which showed that only relatively few vibrations are excited by the $\pi^{*}$ shape resonance, despite the large number of normal modes, indicating a high selectivity of the excitation process. An attempt was made to rationalize the high selectivity by defining selection rules, the initial formulation of which stated that for a shape resonance with a spatial symmetry $\Gamma_{r}$, the vibrational modes that give non-zero element,

$$
\left\langle\Gamma\left(\chi_{1}\right)\left|\left[\Gamma_{r}^{2}\right]\right| \Gamma\left(\chi_{0}\right)\right\rangle,
$$

can be excited. The $\left[\Gamma_{r}^{2}\right]$ is used as a symmetric part of the square of the representation $\Gamma_{r}$, and $\Gamma\left(\chi_{0}\right)$ and $\Gamma\left(\chi_{1}\right)$ are symmetries of initial and final vibrational functions. Here, we consider $\Gamma\left(\chi_{0}\right)$ equal $A_{1}^{\prime}$ (symmetric ground vibrational state), and $\Gamma\left(\chi_{1}\right)$ corresponds to the first excited state which inherits the symmetry of the vibrational mode under examination. The procedure to find the symmetric and anti-symmetric parts of the squared irreducible representations can be found in the literature. ${ }^{27,54}$ Wong and Schulz noted, however, that this initial formulation of the rules is too restrictive because the experimental spectrum showed excitation of several nodes, namely, out-of-plane bending vibrations, which would be forbidden by the simple rule. They then noted that all these extra modes could be explained with the additional assumption that the outgoing electron may also leave in an $s$-wave in which case selection rule (16) was extended ${ }^{26,27}$ by an exception that resonant vibrational excitation may also be expected for modes with the symmetry $\Gamma_{r}$.

\section{A. Excitation of the $A_{1}^{\prime}$ totally symmetrical vibrational mode $v_{3}$}

The present DMR model enables the explicit calculation of values of the transition elements for all incoming and outgoing partial waves and thus allows insight into the applicability of the symmetry selection rules. The squared elements $\left|T_{l m}^{l^{\prime} m^{\prime}}\right|^{2}$ for the symmetric stretch mode $v_{3}$ are shown as vertical columns for all combinations of the incoming and outgoing partial waves up to $l_{\max }=3$ in the left panel of Figure 9. The fact that a single column dominates the figure demonstrates clearly that the resonant vibrational excitation of the $v_{3}$ mode is driven by a single partial wave, which is the lowest partial wave of $A_{2}^{\prime}$ symmetry. With the carbon ring of the molecule placed in the $x y$ plane and one carbon atom on the $y$-axis, we may identify this partial wave as $l=3$, $m=3$. This single contribution to the inelastic cross section is diagonal and as such it describes a collision in which an incoming $A_{2}^{\prime}$ wave excites the fully symmetric ring stretch mode $v_{3}$ and leaves the system in the same partial wave, $A_{2}^{\prime}$. In the right panel of Fig. 9, we display the calculated differential cross section and the spatial shape of the corresponding virtual orbital of $A_{2}^{\prime}$ symmetry. It is clear that the angular shape of the differential cross section describes an almost pure $f$-wave scattering event in agreement with what our analysis shows in the left panel of Fig. 9.

Application of the selection rules to this event leads to the symmetry element

$$
\left\langle A_{1}^{\prime}\left|\left[A_{2}^{\prime 2}\right]\right| A_{1}^{\prime}\right\rangle=\left\langle A_{1}^{\prime}\left|\left[A_{1}^{\prime}\right]\right| A_{1}^{\prime}\right\rangle=\left\langle A_{1}^{\prime}\left|A_{1}^{\prime}\right| A_{1}^{\prime}\right\rangle \neq 0
$$

and the excitation of the $v_{3}$ vibration via the $5.5 \mathrm{eV} A_{2}^{\prime}$ resonance is thus in accord with the selection rules. ${ }^{26,27}$

\section{B. Excitation of the $A_{1}^{\prime \prime}$ vibrational mode $\boldsymbol{v}_{4}$}

The excitation of the $\mathrm{HCH}$ twist mode $v_{4}$ is forbidden by the symmetry selection rules of Wong and Schulz ${ }^{26}$ and Gallup ${ }^{27}$ since both the element for the primary selection rule

$\left\langle A_{1}^{\prime \prime}\left|\left[A_{2}^{\prime 2}\right]\right| A_{1}^{\prime}\right\rangle=\left\langle A_{1}^{\prime \prime}\left|\left[A_{1}^{\prime}\right]\right| A_{1}^{\prime}\right\rangle=\left\langle A_{1}^{\prime \prime}\left|A_{1}^{\prime}\right| A_{1}^{\prime}\right\rangle=0$

and for the $s$-wave exception 

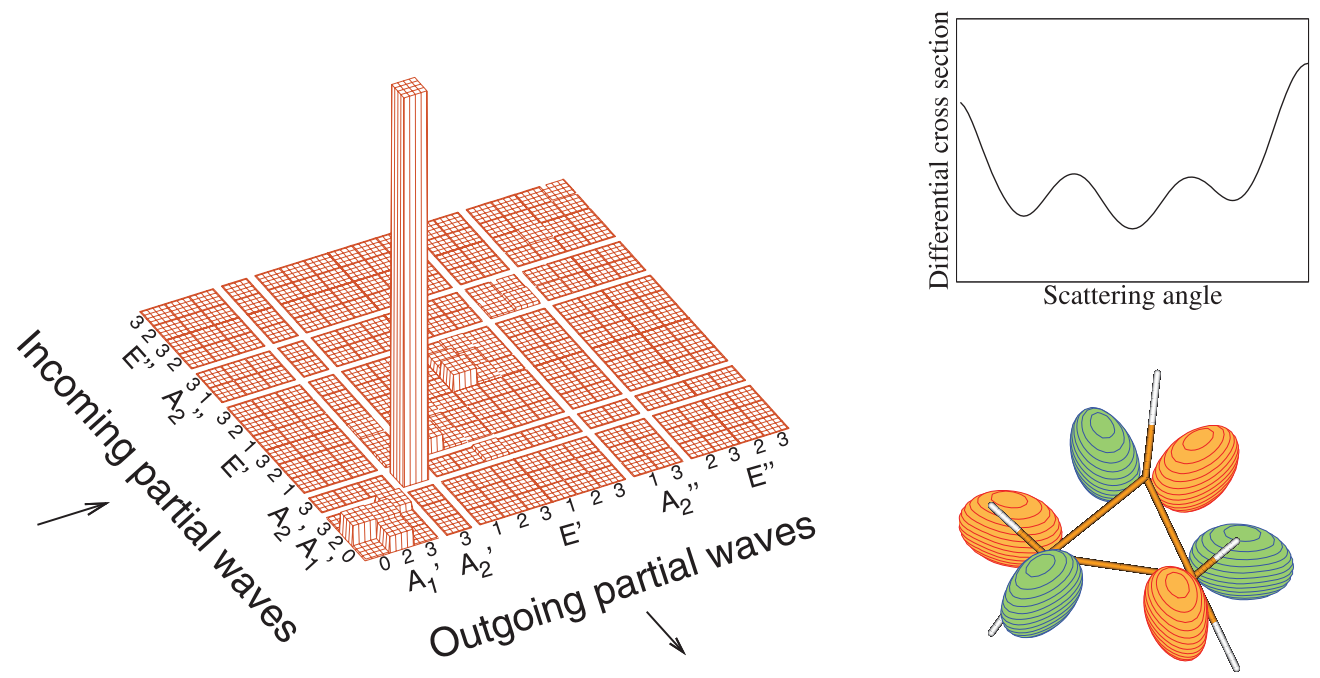

FIG. 9. Symmetric $\mathrm{C}-\mathrm{C}$ stretch $v_{3}$. The left panel displays the squared elements $\left|T_{l m}^{l^{\prime} m^{\prime}}\right|^{2}$ for vibrational excitation of the ring stretch mode $v_{3}$ calculated via Eq. (15). Partial waves up to $l_{\max }=3$ are separated into irreducible representations of the $\mathrm{D}_{3 \mathrm{~h}}$ point group. The integer numbers closest to the graph represent the angular quantum number $l$ of each angular function belonging to the symmetry component. The right panel displays shape of computed differential cross section (upper picture) and shape of a virtual orbital corresponding to outgoing wave of $A_{2}^{\prime}$ symmetry (lower picture).

$$
\left\langle A_{1}^{\prime \prime}\left|\left[A_{2}^{\prime}\right]\right| A_{1}^{\prime}\right\rangle=\left\langle A_{1}^{\prime \prime}\left|A_{2}^{\prime}\right| A_{1}^{\prime}\right\rangle=0
$$

are zero. In contrast to this, the excitation of the $\mathrm{HCH}$ twist mode $v_{4}$ is strong, in particular in the $90^{\circ}$ spectrum. This is seen in the calculated spectrum in Fig. 6 and is confirmed by the experiment, despite the fact that the two vibrations $v_{3}$ and $v_{4}$ are not fully resolved. The first experimental indication is that the $90^{\circ}$ dip in the angular distribution measured at the energy loss of $147 \mathrm{meV}$ (Fig. 2 of Ref. 8) is less deep than a pure $f$-wave, which would be expected for the excitation of $v_{3}$ alone. A second indication is a small $(\sim-4 \mathrm{meV})$ downward shift of the energy-loss peak observed at $\sim 147 \mathrm{meV}$ when recorded at $90^{\circ}$ (when $v_{4}$ dominates) as opposed to when recorded at $135^{\circ}$ or $180^{\circ}$ (when $v_{3}$ dominates) (see Fig. 3 for

the $90^{\circ}$ spectrum and Fig. 1 of Ref. 8 for the $135^{\circ}$ spectrum). The shift is observable despite the fact that the difference of the $v_{3}(146.7 \mathrm{meV})$ and $v_{4}(139.6 \mathrm{meV})$ frequencies is only $7 \mathrm{meV}$, less than the experimental resolution of $15 \mathrm{meV}$.

A comparison of the squared elements $\left|T_{l m}^{l^{\prime} m^{\prime}}\right|^{2}$ for the $v_{4}$ mode (Fig. 10) with those for the totally symmetrical mode $v_{3}$ in Fig. 9 reveals that the resonant excitation mechanism is quite different. The coupling between incoming and outgoing partial waves is off-diagonal. Figure 10 shows that the resonant vibrational excitation of the $v_{4}$ mode is caused by the incoming partial wave $(l=3, m=3)$ of the $A_{2}^{\prime}$ symmetry as in the previous case of the $v_{3}$ mode. However, now the electron causes excitation of the non-symmetric $\mathrm{HCH}$ twisting mode $v_{4}$ of the $A_{1}^{\prime \prime}$ symmetry and leaves the molecule dominantly in two
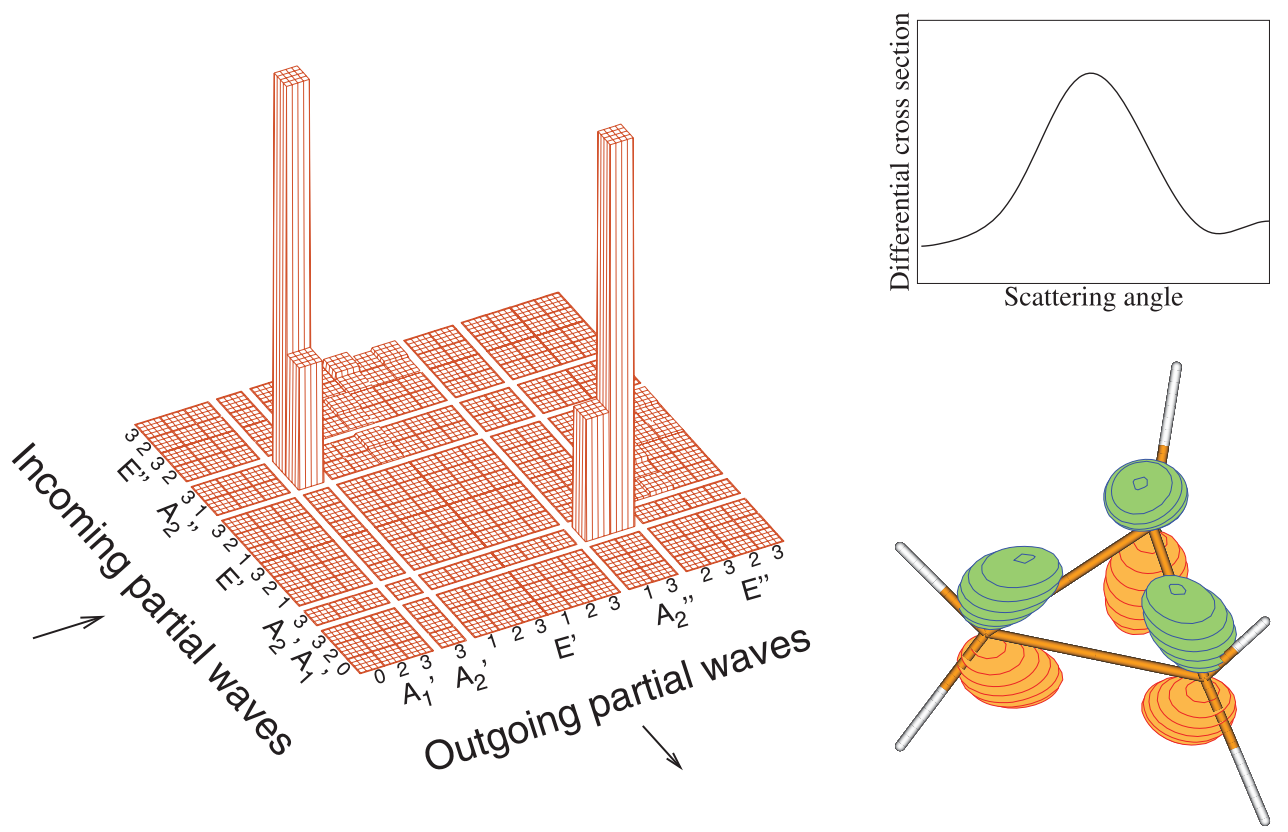

FIG. 10. HCH twist mode $v_{4}$. The left panel displays the squared elements $\left|T_{l m}^{l^{\prime} m^{\prime}}\right|^{2}$ for vibrational excitation of the HCH twist mode $v_{4}$ calculated via Eq. (15). The right panel displays the shape of the computed differential cross section (upper picture) and the shape of a virtual orbital corresponding to the outgoing wave of the $A_{2}^{\prime \prime}$ symmetry (lower picture). 
different partial waves, $l=1, m=0$ and $l=3, m=0$, shown as a pair of adjacent columns in the left panel of Fig. 10. Note that in the angular momentum space limited to $l \leq 3$ used here, and for a given orientation of the molecule, these two partial waves form exactly the irreducible representation $A_{2}^{\prime \prime}$. The fact that there are two pairs of columns, arranged symmetrically across the diagonal, is due to the principle of microscopic reversibility, i.e., the symmetries of the incoming and outgoing waves can be switched.

What we observe here is an $f$-wave resonance of $A_{2}^{\prime}$ symmetry that leaks partially to a $p$-wave in $A_{2}^{\prime \prime}$ symmetry while causing resonant vibrational excitation of non-symmetric $v_{4}$ mode of $A_{1}^{\prime \prime}$ symmetry. The dominant contribution of the corresponding partial waves and the angular shape of differential cross section (both displayed in Fig. 10) together with strength of the resonance (shown in Fig. 5) confirm that it is not a weak effect. The possibility of an outgoing $p$-wave has not been foreseen in the formulation of the resonant selection rules, ${ }^{26,27}$ which thus cease to be valid. Note that extending the selection rules for an outgoing $p$ or even higher $l$ waves is not very meaningful because too many excitations would become allowed and the predictive power would suffer. The present method is superior in that it allows a quantitative prediction of the strength of each individual excitation mechanism.

\section{SUMMARY}

We present a very comprehensive quantitative comparison of the experimental cross section with the results of the recent DMR theory, comprising the energy range from 0.4 to $20 \mathrm{eV}$, the scattering angle range from $10^{\circ}$ to $180^{\circ}$, and all normal modes. An excellent agreement is observed for collision energies under $10 \mathrm{eV}$ and proves the capacity of the DMR theory to quantitatively describe the vibrational excitation process and thus, in a broader sense, the coupling of electronic and nuclear motions in temporary negative ions, with the great advantage of being applicable even to large polyatomic molecules. For the collision energies above $10 \mathrm{eV}$, the DMR method tends to overestimate absolute cross sections in both elastic and inelastic channels.

We believe that the present form of the DMR theory describes quantitatively three important aspects of the process, the mode selectivity for all the vibrational modes, as revealed by the good agreement with the electron-energy loss spectra, the energies and widths of the resonances, as revealed by the good agreement of the cross sections measured at a given energy loss as a function of the electron energy, and the angular distributions, informative of the symmetry aspects.

The importance of taking into account the overlap of close-lying vibrations, not fully resolved experimentally, is emphasized. Treating this aspect properly permitted us to reconcile two absolute measurements ${ }^{8,50}$ which were seemingly in contradiction. The difference of the two experimental results is shown to be entirely explained by the different resolutions and nominal energy-loss values of the two experiments and thus different degrees of vibrational band overlap.

The $5.5 \mathrm{eV} A_{2}^{\prime}$ resonance was used to study the role of symmetry in the mode selectivity with reference to the symmetry selection rules derived by Wong and Schulz ${ }^{26}$ and Gallup. ${ }^{27}$ Detailed insight into this question is gained by calculating the squared transition elements $\left|T_{l m}^{l^{\prime} m^{\prime}}\right|^{2}$ for a given vibration and all combinations of the incoming and outgoing partial waves, and plotting them in a $3 \mathrm{D}$ diagram. This procedure was applied to excitation of the $v_{3}$ totally symmetrical $\mathrm{C}-\mathrm{C}$ stretch mode where a single dominant partial wave $l=3$ of $A_{2}^{\prime}$ symmetry was found. This result is in agreement with the conclusions previously published in the literature ${ }^{21,22}$ and it conforms to the symmetry selection rules. ${ }^{26,27}$ The calculations, confirmed by experiment, have further revealed the excitation of the non-totally symmetrical $\mathrm{HCH}$ twist mode $v_{4}$ that also exhibits a strong resonant behavior at $5.5 \mathrm{eV}$ and that is unexpected because it is forbidden by the symmetry selection rules. In this case, we identified the mechanism by which an $f$-wave resonance of $A_{2}^{\prime}$ symmetry (the same as in the above case of $v_{3}$ ) leaks partially to a $p$-wave in the $A_{2}^{\prime \prime}$ symmetry. This change of electronic symmetry during the collision is mediated by the resonant excitation of the non-symmetric $\mathrm{HCH}$ twisting mode $v_{4}$. This inelastic scattering event shows that the symmetry selection rules as generally formulated, i.e., which assume that the partial wave of the electron either does not change in the process of the inelastic scattering or that it changes such that the electron departs in an $s$-wave, are not sufficiently general and do not explain the observations for cyclopropane. The present theory allows to calculate quantitatively the transition matrix elements for all incoming and departing electron partial waves and is thus more powerful than the selection rules. Note that excitation of non-totally symmetrical modes often plays a crucial role in electron-driven chemistry (dissociative electron attachment) because the ensuing symmetry lowering offers a pathway to by-pass an energy barrier. However, it has not been conclusively proven that the symmetry lowering is required to by-pass an energy barrier-but the experimental evidence certainly indicates a propensity to lower the symmetry. The dissociative electron attachment of $\mathrm{CF}_{4}$ is a good example. ${ }^{55}$

\section{ACKNOWLEDGMENTS}

This work was conducted within the framework of the COST Action CM1301 (CELINA). We acknowledge the support of the Czech Ministry of Education (Grant No. LD14088), the Grant Agency of the Czech Republic (Grant No. P208/11/0452), and the Swiss National Science Foundation, Project No. 200020-144367.

${ }^{1}$ S. Mazevet, M. A. Morrison, O. Boydstun, and R. K. Nesbet, Phys. Rev. A 59, 477 (1999).

${ }^{2}$ H. Feng, W. G. Sun, and M. A. Morrison, Phys. Rev. A 68, 062709 (2003).

${ }^{3}$ C. H. Greene and Ch. Jungen, Phys. Rev. Lett. 55, 1066 (1985).

${ }^{4}$ K. Houfek and M. Čížek, in Low-Energy Electron Scattering from Molecules, Biomolecules and Surfaces, edited by P. Čársky and R. Čurík (CRC Press, New York, 2012).

${ }^{5}$ T. N. Rescigno, W. A. Isaacs, A. F. Orel, H. D. Meyer, and C. W. McCurdy, Phys. Rev. A 65, 032716 (2002).

${ }^{6}$ D. J. Haxton, Z. Zhang, H. D. Meyer, T. N. Rescigno, and C. W. McCurdy, Phys. Rev. A 69, 062714 (2012).

${ }^{7}$ R. Čurík and P. Cársky, J. Phys. B 36, 2165 (2003).

${ }^{8}$ R. Čurík, P. Čársky, and M. Allan, Phys. Rev. A 86, 062709 (2012).

${ }^{9}$ P. Čársky, Int. J. Quantum Chem. 109, 1237-1242 (2009).

${ }^{10} \mathrm{P}$. Čársky, R. Čurík, and Š. Varga, J. Chem. Phys. 136, 114105 (2012). 
${ }^{11}$ P. Čársky and R. Čurík, Theor. Chem. Acc. 133, 1466 (2014).

${ }^{12}$ J. Tennyson, Phys. Rep. 491, 29 (2010).

${ }^{13}$ Z. Masin and J. D. Gorfinkiel, J. Chem. Phys. 135, 144308 (2011).

${ }^{14}$ T. N. Rescigno, C. W. McCurdy, A. E. Orel, and B. H. Lengsfield III, in Computational Methods for Electron-Molecule Collisions, edited by W. M. Huo and F. A. Gianturco (Plenum Press, New York, 1995).

${ }^{15}$ C. S. Trevisan, A. E. Orel, and T. N. Rescigno, Phys. Rev. A 74, 042716 (2006).

${ }^{16}$ K. Takatsuka and V. McKoy, Phys. Rev. Lett. 45, 1734 (1980).

${ }^{17}$ P. Palihawadana, J. Sullivan, M. Brunger, C. Winstead, V. McKoy, G. Garcia, F. Blanco, and S. Buckman, Phys. Rev. A 84, 062702 (2011).

${ }^{18}$ M. A. Khakoo, J. Muse, K. Ralphs, R. F. da Costa, M. H. F. Bettega, and M. A. P. Lima, Phys. Rev. A 81, 062716 (2010).

${ }^{19}$ F. A. Gianturco, D. G. Thompson, and A. Jain, in Computational Methods for Electron-Molecule Collisions, edited by W. M. Huo and F. A Gianturco (Plenum Press, New York, 1995).

${ }^{20}$ I. Baccarelli, I. Bald, F. A. Gianturco, E. Illenberger, and J. Kopyra, Phys. Rep. 508, 1 (2011).

${ }^{21}$ M. Allan and L. Andric, J. Chem. Phys. 105, 3559 (1996).

${ }^{22}$ R. Čurík and F. A. Gianturco, J. Phys. B 35, 1235 (2002).

${ }^{23}$ M. Allan, J. Am. Chem. Soc. 115, 6418 (1993).

${ }^{24}$ T. Beyer, B. M. Nestmann, B. K. Sarpal, and S. D. Peyerimhoff, J. Phys. B 30, 3431 (1997).

${ }^{25}$ H. Tawara, Y. Itikawa, H. Nishimura, H. Tanaka, and Y. Nakamura, National Institute for Fusion Science, NIFS-DATA-6, 1990.

${ }^{26}$ S. F. Wong and G. J. Schulz, Phys. Rev. Lett. 35, 1429 (1975).

${ }^{27}$ G. A. Gallup, J. Chem. Phys 99, 827 (1993).

${ }^{28}$ M. Allan, J. Phys. B 40, 3531 (2007).

${ }^{29}$ M. Allan, Phys. Rev. A 81, 042706 (2010).

${ }^{30} \mathrm{P}$. Čársky and R. Čurík, in Computational Chemistry: Reviews of Current Trends, edited by J. Leszczynski (World Scientific, 2006), Vol. 10.

${ }^{31}$ P. Čársky and R. Čurík, in Low-Energy Electron Scattering from Molecules, Biomolecules and Surfaces, edited by P. Č́rsky and R. Čurík (CRC Press, New York, 2012).

${ }^{32}$ C. J. Joachain, Quantum Collision Theory (Elsevier Science Publishers B.V., Amsterdam, 1983), pp. 577-621.

${ }^{33}$ J. P. Perdew and A. Zunger, Phys. Rev. B 23, 5048 (1981).

${ }^{34}$ R. Čurík and M. Šulc, J. Phys. B 43, 175205 (2010).
${ }^{35}$ T. H. Dunning, Jr. and P. J. Hay, in Modern Theoretical Chemistry, edited by H. F. Schaefer III (Plenum, New York, 1977).

${ }^{36}$ Z. Rinkevicius, I. Tunnel, P. Salek, O. Vahtras, and A. Hans, J. Chem. Phys. 119, 34 (2003).

${ }^{37}$ J. Sadlej, Collect. Czech. Chem. Commun. 53, 1955 (1988).

${ }^{38}$ S. V. Krishtenko, A. I. Maslov, and V. Shevelko, Molecules and Their Spectroscopic Properties (Springer, Berlin, 1998).

${ }^{39}$ H. Nishimura and H. Tawara, J. Phys. B 24, L363 (1991).

${ }^{40}$ C. Makochekanwa, H. Kato, M. Hoshino, H. Tanaka, H. Kubo, A. R. Lopes,

M. A. P. Lima, and L. G. Ferreira, J. Chem. Phys. 124, 024323 (2006).

${ }^{41}$ C. Szmytkowski and S. Kwitnewski, J. Phys. B 35, 2613 (2002).

${ }^{42}$ R. Čurík and F. A. Gianturco, J. Phys. B 35, 717 (2002).

${ }^{43}$ C. Winstead, Q. Sun, and V. McKoy, J. Chem. Phys. 96, 4246 (1992).

${ }^{44}$ See supplementary material at http://dx.doi.org/10.1063/1.4917304 for larger data set of elastic and inelastic cross sections for cyclopropane.

${ }^{45}$ NIST Chemistry WebBook, edited by P. J. Linstrom and W. G. Mallard, NIST Standard Reference Database Vol. 69 (National Institute of Standards and Technology, Gaithersburg, MD, 2011), p. 20899, http://webbook.nist.gov.

${ }^{46}$ M. B. Robin, Higher Excited States of Polyatomic Molecules (Academic Press Inc. (London) Ltd., 1985), Vol. III, p. 101.

${ }^{47}$ C. Benesch, M. Č́́žek, M. Thoss, and W. Domcke, Chem. Phys. Lett. 430, 355 (2006).

${ }^{48}$ C. Benesch, M. Čížek, J. Klime, I. Kondov, M. Thoss, and W. Domcke, J. Phys. Chem. C 112, 9880 (2008).

${ }^{49}$ A. E. Howard and S. W. Staley, ACS Symp. Ser. 263, 183 (1984).

${ }^{50}$ H. Kato, M. Hoshino, H. Kawahara, C. Makochekanwa, S. J. Buckman, M. J. Brunger, H. Cho, M. Kimura, D. Kato, H. A. Sakaue, I. Murakami, T. Kato, and H. Tanaka, National Institute for Fusion Science, NIFS-DATA$105,2009$.

${ }^{51}$ C. Makochekanwa, H. Kato, M. Hoshino, H. Cho, M. Kimura, O. Sueoka, and H. Tanaka, Eur. Phys. J. D 35, 249 (2005).

${ }^{52}$ V. I. Lebedev and D. N. Laikov, Dokl. Akad. Nauk 366, 741 (1999).

${ }^{53}$ S. L. Altmann and C. J. Bradley, Philos. Trans. R. Soc. London A 255, 199 (1963).

${ }^{54}$ L. D. Landau and E. M. Lifshitz, Quantum Mechanics, Non-Relativistic Theory, 3rd ed. (Pergamon Press, Oxford, 1977), p. 370, Sec. \$94.

${ }^{55}$ F. H. Ómarsson, E. Szymanska, N. J. Mason, E. Krishnakumar, and O. Ingólfsson, Phys. Rev. Lett. 111, 063201 (2013). 